\title{
Image Registration in Myocardial Perfusion MRI
}

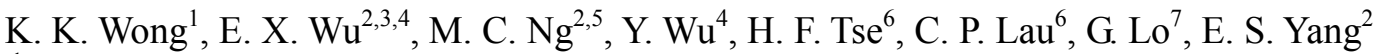 \\ ${ }^{1}$ HCNR Center of Bioinformatics and Department of Radiology, Brigham and Women's Hospital, \\ Harvard Medical School, Boston, MA, USA \\ ${ }^{2}$ Jockey Club MRI Centre, \\ ${ }^{3}$ Department of Electrical and Electronic Engineering, \\ ${ }^{4}$ Department of Medical Engineering, \\ ${ }^{5}$ Department of Orthopedics and Traumatology, \\ ${ }^{6}$ Department of Medicine, \\ University of Hong Kong, Pokfulam, Hong Kong \\ ${ }^{7}$ Hong Kong Sanatorium Hospital, Hong Kong
}

\begin{abstract}
In clinical first-pass myocardial perfusion studies, physiological and patient motions are inevitable. Such motions impair the sensitivity and reliability in assessing myocardial perfusion abnormalities. The current study aims to correct the misregistration of myocardium during first-pass perfusion imaging by using a normalized mutual information approach. Multi-slice registrations were performed in 15 sets of clinical data sets. The proposed registration procedure was found to be fairly robust. With such registration, ROI time courses from normal myocardium and infarcted myocardium were improved, offering potential to quantify myocardial perfusion parameters in a more reliable manner.
\end{abstract}

\section{INTRODUCTION}

Motion always appears in one form or another in clinical MRI. Physiological and patient motions are detrimental in time series imaging as it interferes with the temporal signal analysis. Therefore it is desirable to correct for the motion by image registration before time domain analysis. In first-pass myocardial perfusion imaging, image registration become more complex as the motion are usually non-rigid in nature and incoherent motions among different organs and tissues are common. In addition, contrast inflow invalidates most traditional registration methods as the assumption of similarity is violated. Alternative methods have been proposed [1-4], most of which involve intensity thresholding to modify the image before registering with traditional methods. However, the success of this approach varies from site to site and therefore it is protocol dependent. In patients with cardiac disease, unpredictable intensity change associated with turbulence flow in the cardiac chamber would be expected. In this paper, a method based on normalized mutual information (NMI) approach [5] is proposed to automatically correct for multi-slice image misregistration without intensity thresholding. The aim is to obtain more accurate perfusion parameters and improve sensitivity in detection of myocardial perfusion abnormalities.

\section{METHODS}

Fifteen patients were scanned in a previously reported study of myocardial therapeutic interventions [6]. Total 30 scans were done using a GE Signa 1.5T MRI system (GE Medical Systems, Milwaukee, MI) with a twin gradient subsystem. First-pass dobutamine stressed perfusion images were acquired in every heartbeat during bolus injection of exogenous contrast agent (Gd-DTPA). Images were acquired for 1 minute using fast gradient echo sequence. For coregistration, all images were first smoothed by a $3 \mathrm{~mm}$ FWHM Gaussian kernel and a simple rectangular image mask was used to include the heart to test the robustness of the algorithm. Image registration was achieved by maximizing the NMI of pair-wise images from consecutive scans. NMI of pair-wise images, $\mathrm{M}_{\mathrm{i}}$ and $\mathrm{M}_{\mathrm{i}+1}$, are defined as:

$$
\mathrm{NMI}=\left[\mathrm{H}\left(\mathrm{M}_{\mathrm{i}}\right)+\mathrm{H}\left(\mathrm{M}_{\mathrm{i}+1}\right)\right] / \mathrm{H}\left(\mathrm{M}_{\mathrm{i}}, \mathrm{M}_{\mathrm{i}+1}\right) .
$$

where $H\left(M_{i}\right), H\left(M_{i+1}\right)$ are the marginal entropies of $M_{i}$ and $M_{i+1}$ respectively, and $\mathrm{H}\left(\mathrm{M}_{\mathrm{i}}, \mathrm{M}_{\mathrm{i}+1}\right)$ is the joint entropy of the pair-wise images. Relative in-plane translations were applied to the images within a \pm 3 search window until a match was found. The scales of the search window were varied from 1-pixel, to 0.5-pixel, 0.25-pixel, 0.125-pixel, 0.0625-pixel consecutively to achieve sub-pixel image registration. The results were evaluated by plotting the signal change across the time series on the two orthogonal planes intersected at the center of left ventricle in each individual slice.

\section{RESUlts}

The myocardial perfusion images are registered satisfactorily in both small motion and large motion cases. In the case of ECG mis-triggering, the algorithm was able to reduce the misregistration due to translation and is fairly robust. Representative examples of a small misregistration case and a large misregistration case are shown in Fig. 1 and Fig. 2 respectively. Fig. 1a shows the slice under investigation and the arrows indicates the intensity profile location. Fig $1 \mathrm{~b}$ plots the original intensity profile on horizontal axis and shows the intensity change with contrast injection on the vertical axis. After registration, misregistration was reduced and the papillary muscle was consistently seen across all time points as indicated by the small arrow in Fig. 1c. Similarly, Fig 2a shows the location of intensity profile. Fig. $2 \mathrm{~b}$ shows the original large intensity change before registration during a 30 time-point scan. Fig. 2c shows the registration result with apparent improvement in the intensity profile in the left ventricle and also in the lateral and interseptal walls. Note that residual movement of other organs and chest wall are seen, showing incoherent motions with respect to the heart position. For illustration, one slice of the case in Fig. 2 was investigated with an ROI analysis. Two ROIs were placed on healthy myocardium on the septal and lateral walls as shown in the upper two circles in Fig 3a. The lower circular ROI in Fig 3a corresponds to the site of myocardial infarct, which was hyperintense in the 15-minute delayed enhancement image as in Fig. 3b. The time courses from the three ROIs were compared before and after image registration. Fig. 3c shows the ROI time courses from the original dataset. From the figure, it was difficult to assess and quantify the perfusion defect due to motion-induced image misregistration. Fig. $3 \mathrm{~d}$ shows the translations registered version of which the ROI time courses from the septal and lateral wall were much more consistent in terms of time to peak and contrast washout. The low perfusion defect can be immediately detected after the misregistration had been corrected using the proposed co-registration approach. 

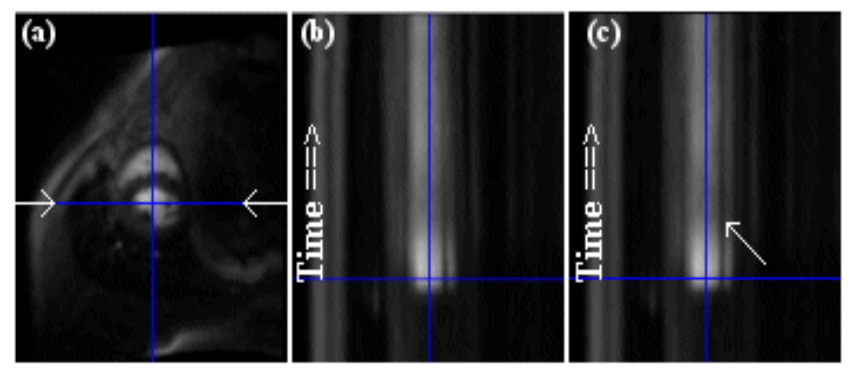

Fig. 1. Short-axis cut of the heart (a) during contrast arrival. The profile intensity-time relationship (b) before registration and (c) after registration is shown.
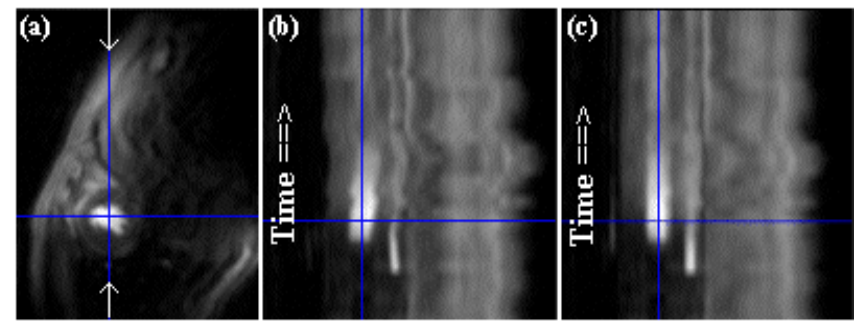

Fig. 2. Short-axis cut of the heart (a) during contrast arrival. The profile intensity-time relationship (b) before registration and (c) after registration is shown.
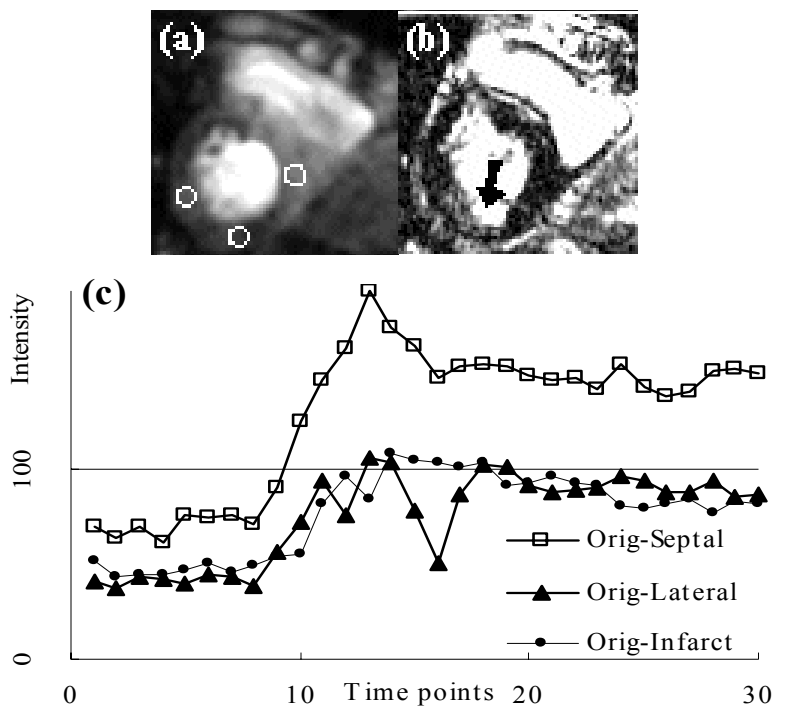

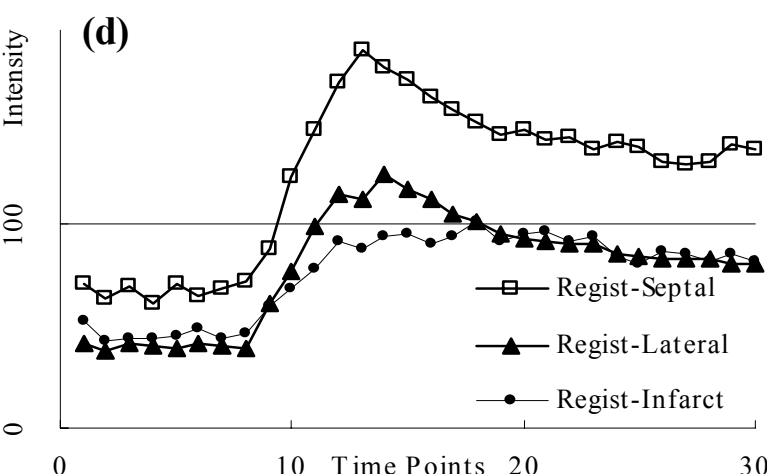

Fig. 3. Three ROIs are placed on healthy septal and lateral walls and also on the lateral infarct myocardium as shown in (a). The location of infarct is marked on the (b) 15-minute delayed enhancement image. ROI signal time courses are plotted for the (c) original data and the (d) post registration data.

\section{DISCUSSION AND CONCLUSION}

A novel method is proposed to automatically correct for multi-slice image misregistration based on normalized mutual information. This method does not depend on intensity thresholding, therefore is more robust. Preliminary results have demonstrated much improved ROI time courses among various myocardial areas in presence of patient motions. In patient cardiac MRI study, such coregistration is expected to provide more reliable and quantitative assessment of myocardial perfusion parameters. More validation and optimization are underway.

\section{ACKNOWLEDGEMENT}

The author would like to thanks the Jockey Club Charities Trust for the funding support. This work was done in the Jockey Club MRI Center, University of Hong Kong.

\section{REFERENCES}

[1] S.N. Gupta, M. Solaiyappan, G..M. Beache, A.E. Arai, T.K. Foo. Fast method for correcting image misregistration due to organ motion in time-series MRI data. Magn Reson Med, vol 49, pp. 506-514, Mar 2003

[2] C.M. Gallippi, C.M. Kramer, Y.L. Hu, D.A. Vido, N. Reichek, W.J. Rogers. Fully automated registration and warping of contrastenhanced first-pass perfusion images. J Cardiovasc Magn Reson, vol. 4, pp. 459-469, 2002.

[3] T. Delzescaux, F. Frouin, A. de Cesare, S. Philipp-Foliquet, A. Todd-Pokropek, A. Herment, M. Janier. Using an adaptive semiautomated self-evaluated registration technique to analyze MRI data for myocardial perfusion assessment. J Magn Reson Imaging., vol. 18, pp. 681-90, Dec 2003.

[4] L.M. Bidaut, J.P. Vallee. Automated registration of dynamic MR images for the quantification of myocardial perfusion. J Magn Reson Imaging, vol. 13, pp 648-655, Apr 2001.

[5] C. Studholme, E. Novotny, I.G. Zubal, J.S. Duncan. Estimating tissue deformation between functional images induced by intracranial electrode implantation using anatomical MRI. Neuroimage, vol. 13, pp. 561-576, Apr 2001.

[6] H.F. Tse, Y.L. Kwong, J.K. Chan, G. Lo, C.L. Ho, C.P. Lau. Angiogenesis in ischaemic myocardium by intramyocardial autologous bone marrow mononuclear cell implantation. Lancet, vol. 361, pp 47-49, Jan 2003. 